\title{
Identifying Factors That Predict Worse Constipation Symptoms in Palliative Care Patients: A Secondary Analysis
}

\author{
Katherine Clark, MB, BS, MMed, FRACP, ${ }^{1,2}$ Lawrence T. Lam, PhD, BSc, \\ Nicholas J. Talley, MD, PhD, FRACP, Jane L. Phillips, RN, PhD, and David C. Currow, PhD, MPH, FRACP
}

\begin{abstract}
Objective: The aim of this work was to investigate whether variables identified as likely to impact the experience of constipation in other clinical settings similarly affected the experiences of constipated palliative care patients. Background: The majority of palliative care patients with cancer are likely to be bothered by constipation symptoms at some point in their disease trajectory. Despite this, it remains unclear as to which factors predict more severe problems.

Methods: This study was conducted in a sample of 94 constipated palliative care patients who were asked to voluntarily complete a series of questions regarding their demographic and other characteristics, including whether they had chronic constipation symptoms, that is, constipation symptoms for 12 months. Other variables included age, body mass index, sex, performance status, and regular opioids and their doses. At the same time, they were asked to complete the Patient Assessment of Constipation Symptoms (PAC-SYM) and Patient Assessment of Constipation Quality of Life (PAC-QOL) questionnaires.

Results: Descriptive statistics summarized baseline data. Unadjusted associations between the selected variables on PAC-SYM were examined by using bi-variate analyses. Significant variables identified on bi-variate analyses were included in a multivariate analysis. The final results identified that only the chronicity of constipation symptoms predicted more severe symptoms. This relationship persisted when this single variable was retained in the final model, illustrating that PAC-SYM scores are 0.41 higher in patients with chronic constipation compared with those without it $(p=0.02)$. In contrast, regular opioid use was not identified as a significant factor $(p=0.56)$.

Discussion: This study suggests that the factor most likely to predict worse constipation symptoms was the duration that people had experienced problems. Further, those who perceived their constipation symptoms to be more severe had a poorer quality of life. More work is required to better define constipation risk factors and ways to best modify a patient's experiences.
\end{abstract}

Keywords: palliative care; constipation symptoms; severity

\section{Introduction}

$\mathbf{C}$ ONSTIPATION SYMPTOMS are common in palliative care. Point prevalence studies indicate that at any one time point, nearly $20 \%$ of this patient cohort will report moderateto-severe constipation problems. ${ }^{1}$ Further, longitudinal data indicate that the significant majority of people will experience symptoms at some point during their palliative care disease trajectory. ${ }^{2}$ In contrast, $21 \%$ of the general population report having experienced constipation at some point, and only $3 \%$ have experienced longer term chronic symptoms. ${ }^{3}$

The burden of constipation in palliative care is reiterated by the fact that laxatives are among the most commonly prescribed medications for this group. ${ }^{4}$ Despite the prevalence of this symptom, there is no consensus as how best to diagnose constipation or tailor treatments in palliative care.

\footnotetext{
${ }^{1}$ Department of Palliative Care, Calvary Mater Newcastle, Newcastle, Australia.

${ }^{2}$ School of Medicine and Public Health, The University of Newcastle, Newcastle, Australia.

${ }^{3}$ Centre for Cardiovascular and Chronic Care, Faculty of Health, University of Technology Sydney, Sydney, Australia.

${ }^{4}$ Flinders Centre for Clinical Change, Flinders University, Adelaide, Australia.

Accepted September 19, 2016.
} 
Objective data that define whether there are predisposing factors that worsen the severity of a palliative patient's constipation symptoms are lacking.

Given this gap, a secondary analysis of previously collected data was conducted with the aim of investigating whether there were any associations between people's selfreported current constipation symptoms and the factors identified as potentially contributing to worse constipation in the general population, namely: age, ${ }^{5}$ sex,${ }^{5}$ body mass index (BMI) ${ }^{6}$ functional status, ${ }^{6}$ regular opioids, ${ }^{7}$ and whether experience of constipation symptoms had persisted for 12 months. ${ }^{3}$

\section{Methods}

Secondary analysis of the baseline data collected as part of a cohort study comparing the symptom experiences of palliative cancer patients objectively diagnosed with constipation as measured by colon transit times and anorectal manometry compared with usual palliative constipation management. This study was conducted across six Australian palliative care services over four years (Australia and New Zealand Clinical Trials Registry [ANZCTR] trial number ACTRN12611000705987). The larger study was approved by the participating sites' human ethics committees, with this secondary analysis planned as part of the study's protocol. All participants provided written informed consent.

\section{Participants}

Only cancer inpatients and outpatients already considered constipated by their palliative care team and taking laxatives (regularly and as needed) were included. Other criteria for inclusion were that people needed to be older than 18 years of age, have an Australian-modified Karnofsky Performance Status (AKPS) ${ }^{8}$ of $\geq 40$ at enrolment, be willing and able to complete the questionnaires, not currently or intending to fall pregnant, and, in the opinion of the referring doctor, be well enough to complete the study. Exclusion criteria included short-term reversible constipation attributable to specific etiologies such as hypercalcemia or chemotherapy-induced bowel dysfunction; and a known bowel obstruction or previous bowel surgery that resulted in a reduction of the length of the gastrointestinal tract that may have further affected gut transit times.

\section{Factors previously identified as likely to affect constipation symptoms}

A range of demographic and other factors previously suggested as likely to impact the experience of constipation in the general population were incorporated as baseline data in the larger study. This included:

(i) Demographic factors, including age (in years); sex; BMI; and performance status.

(ii) Whether or not regular opioids were required, with the 24-hour dose of those participants who required opioids summarized as the equivalent oral morphine (EOM) dose. The EOM was calculated by using an existing dose conversion table. ${ }^{9}$ Doses were then stratified as low, moderate, high, or very high doses according to pre-existing definitions. ${ }^{10}$ (iii) Chronicity of constipation symptoms as summarized by the Rome criteria. For this work, a previously proposed modification of the Rome III Criteria ${ }^{3}$ was used, requiring people to have experienced two or more symptoms for 12 months, including (1) $<3$ bowel actions per week; (2) straining on at least $25 \%$ of bowel actions; (3) hard stools at least $25 \%$ of the time; and (4) feeling of an incomplete evacuation at least $25 \%$ of the time. ${ }^{3}$ This modified version has been elsewhere and is only dissimilar to both Rome III and Rome IV because of the fact that symptoms are reported for 12 months compared with the more accepted 3-6 months. ${ }^{11}$

\section{Current constipation symptoms}

Participants' current self-reported constipation symptoms were summarized with the self-reported 12-item Patient Assessment of Constipation Symptoms (PAC-SYM). ${ }^{12}$ This validated tool uses a $0-4$ scale (0: absent to 4 : worst possible) examining abdominal and rectal symptoms, and difficulties passing bowel actions. The final score is calculated by dividing the total sum of the scores by the number of the questions answered. The lower the final figure, the less symptomatic the person rates themselves to be. This was accompanied by the Patient Assessment of Constipation Quality of Life (PAC-QOL), ${ }^{13}$ which specifically examines the impact of people's perceptions of their constipation symptoms on quality of life by using an identical scoring format to the PAC-SYM.

\section{Analysis}

Data were analyzed by using STATA SE 10 statistical software (Stata Statistical Software; StataCorp., College Station, TX). Descriptive statistics summarized baseline data. Unadjusted associations between the selected variables on PAC-SYM were examined by using bi-variate analyses. Significant variables identified on bi-variate analyses were included in a multivariate analysis.

\section{Results \\ Patient characteristics}

There were 94 participants who completed some or all of the baseline questions (Table 1). They had a mean age of 67.5 (standard deviation [SD] \pm 15.7$)$ years, and $58.5 \%(n=55)$ were men. Only $3.9 \%(n=3)$ identified themselves as using laxatives on an "as needed" basis, with $97.5 \%(n=90)$ taking at least one type of laxative every day. The majority of the group was using regular opioid analgesia $(n=71)$, with $41.1 \%(n=30)$ on low opioid doses (defined as less than a daily EOM dose of $60 \mathrm{mg}$ ). The remainder were using either moderate doses as defined by an EOM dose of 61-200 mg $(n=26)$ or high to very high doses defined by an EOM of $201 \mathrm{mg}$ to greater than $600 \mathrm{mg}(n=17)$. Of the 73 who answered the chronic constipation section, more than half described constipation symptoms for greater than 12 months.

\section{Constipation symptoms}

The mean score of the PAC-SYM and PAC-QOL of the 88 people who completed this section was 1.2 ( $\mathrm{SD} \pm 0.8$; range 
Table 1. Participant Details

\begin{tabular}{lc}
\hline Characteristics & $\begin{array}{c}\text { Frequency }(\%) \\
\text { or mean }(S D)\end{array}$ \\
\hline Age (years) & \\
$<40$ & $2(2.1 \%)$ \\
$41-50$ & $6(6.4 \%)$ \\
$51-60$ & $14(16.9 \%)$ \\
$61-70$ & $31(33.0 \%)$ \\
$>70$ & $38(42.5 \%)$ \\
Not recorded & $3(3.2 \%)$ \\
Sex & \\
Male & \\
Female & $55(58.5 \%)$ \\
Self-reported number of types of standard $19 x a t i v e s ~ u s e d$ \\
daily & $39(41.5 \%)$ \\
As needed & $3(3.9 \%)$ \\
1 & $43(47.5 \%)$ \\
2 & $33(35.1 \%)$ \\
$3+$ & $14(14.9 \%)$ \\
Not recorded & $1(1.1 \%)$
\end{tabular}

Prescribed laxatives

Stimulant plus softener $\quad 40$

Macrogol 22

Rectal 19

Peripheral opioid antagonist 13

Osmotic 13

Stimulant alone

Fibre

Softener alone

7

3

BMI

$<18$

$18-25$

$>25-30$

$>30$

Not recorded

$6(6.4 \%)$

$46(48.9 \%)$

$15(15.9 \%)$

$11(11.7 \%)$

$16(17.1 \%)$

Performance status (AKPS)
100-80
$12(12.7 \%)$
$79-50$
$78(83.0 \%)$
$<50$
$4(4.3 \%)$

Chronically constipated according to the modified

Rome III criteria

Yes

$51(54.3 \%)$

No

$22(23.4 \%)$

Not recorded

$21(22.3 \%)$

Regular opioids

Yes

$73(77.6 \%)$

No

$13(13.8 \%)$

Not recorded

$8(8.5 \%)$

For those on opioids, mean oral morphine equivalents

Low $(<60 \mathrm{mg})$

Moderate (61-200 mg)

High (201-600 mg)

$30(41.1 \%)$

$26(35.6 \%)$

$13(18.8 \%)$

Very high (>600 mg)

$4(5.5 \%)$

PAC-SYM score

$1.2(0.8)$

PAC-QOL score

$1.3(0.6)$

AKPS, Australian-Modified Karnofsky Performance Status; BMI, body mass index; PAC-QOL, Patient Assessment of Constipation Quality of Life; PAC-SYM, Patient Assessment of Constipation Symptoms; SD, standard deviation.
Table 2. Unadjusted Associations Between the

Study Variables and the Patient Assessment of Constipation Symptoms and Patient Assessment of Constipation Quality of Life

\begin{tabular}{|c|c|c|}
\hline \multirow[b]{2}{*}{ Variables } & \multicolumn{2}{|c|}{ Results on association } \\
\hline & $\begin{array}{l}\text { PAC-SYM } \\
\text { F statistic } \\
\text { (p value) }\end{array}$ & $\begin{array}{l}P A C-Q O L \\
\mathrm{~F} \text { statistic } \\
\text { (p value) }\end{array}$ \\
\hline Age (years) & $2.32(0.06)$ & $0.75(0.56)$ \\
\hline Gender & $1.68(0.20)$ & $0.53(0.47)$ \\
\hline BMI & $0.39(0.76)$ & $0.22(0.88)$ \\
\hline Performance status (AKPS) & $0.99(0.37)$ & $0.19(0.83)$ \\
\hline $\begin{array}{l}\text { Chronic constipation } \\
\text { according to modified } \\
\text { Rome III criteria }\end{array}$ & $5.74(0.02)$ & $2.62(0.11)$ \\
\hline Regular opioids & $0.35(0.56)$ & $0.19(0.67)$ \\
\hline Mean oral morphine equivalents & $0.01(0.98)$ & $0.13(0.72)$ \\
\hline
\end{tabular}

$0-3.43$ ) and 1.3 (SD \pm 0.6 ; range $0-2.88$ ), respectively, suggesting that this group was reasonably symptomatic. This statement is based on the fact that previous data report that the mean PAC-SYM of a cohort of functionally constipated patients dissatisfied with their usual treatment was 1.61 (SD $\pm 0.69),{ }^{12}$ with the mean PAC-QOL scores of a similar cohort being $1.85(\mathrm{SD} \pm 0.67){ }^{13}$

\section{Regression analysis}

A bivariate analysis was undertaken to explore the relationship between identified variables and PAC-SYM, with the results summarized in Table 2 . The only significant unadjusted association was between the severity of selfreported symptoms and the chronicity with which people had been experiencing constipation symptoms. This relationship persisted when this single variable was retained in the final model, which shows that PAC-SYM scores are 0.41 higher in patients with chronic constipation than in those without it $(p=0.02)$ (Table 3).

None of the study variables was significantly associated with PAC-QOL. However, PAC-SYM and PAC-QOL were moderately correlated with a coefficient of $0.61(p<0.001)$, suggesting that the more symptomatically constipated patients were, the poorer their perceived quality of life was overall.

\section{Discussion}

The aim of this work was to undertake an investigation of whether or not factors that have been suggested as likely to

\section{Table 3. Results Obtained from the Regression ANALYSES FOR PATIENT ASSESSMENT of Constipation Symptoms}

\begin{tabular}{lcc}
\hline $\begin{array}{l}\text { Variable retained } \\
\text { in the final model }\end{array}$ & $\begin{array}{c}\text { Co-efficient } \\
\text { (standard error) }\end{array}$ & $\begin{array}{c}\text { Significance } \\
\text { (p value) }\end{array}$ \\
\hline $\begin{array}{l}\text { Chronic constipation } \\
\text { according to modified } \\
\text { Rome III criteria }\end{array}$ & $0.41(0.17)$ & $2.40(0.02)$ \\
\hline
\end{tabular}


contribute to the problems of constipation in the general population affected palliative care patients' self-reported constipation symptoms. The main finding was that those with more chronic problems tended to self-report worse constipation symptoms. This is potentially a very important observation for a number of reasons. First, this work questions whether usual assessments and treatments adequately palliate constipation symptoms when people with pre-existing chronic problems are referred to palliative care. Second, this observation suggests that further work is required to more clearly articulate whether sub-groups of constipated patients within palliative care exist. Although more work is needed, it seems reasonable to suggest that such sub-groupings will include the proportion of people who have experienced problems for many years as opposed to those who develop problems secondary to progressive diseases and other issues such as medications. It is important to more clearly articulate this, as it is highly likely that the approaches used to palliate constipation symptoms in these subgroups will be different. However, this remains speculative and much more work is required to more clearly define the subgroups.

Aside from chronicity of symptoms, the only other variable that was close to being of statistical significance was age. This is interesting, as, similar to chronicity of constipation symptoms, age has also been defined as a risk factor in the general population. ${ }^{5}$ It is not clear whether the relationship between older age and constipation is due to changes in the function of the colon or other factors such as frailty and/or polypharmacy. ${ }^{14}$

Neither the use of regular opioids nor the doses at which they were prescribed were identified as variables that affected the severity of people's perceptions of constipation. This observation requires further examination, as it is in contrast to the reported experiences of people using regular opioids for chronic pain. In this latter cohort, it has been identified that people regularly do not comply with their analgesic regime to improve their experiences of constipation. ${ }^{15}$ However, whether this also occurs in palliative care is not known. ${ }^{16}$ Another pertinent finding that requires comment is the observation that the more severe participants' self-reported symptoms were, the worse the patients perceived their quality of life to be as a result of constipation symptoms. Although this is not a new observation, ${ }^{17}$ it does reiterate the seriousness of this issue and reinforce the need to more clearly define factors that contribute to constipation symptoms and how they can best be addressed so that quality of life can be improved.

There are strengths and weaknesses of this work. A significant strength is that this work used the modified Rome III criteria to summarize the chronicity of problems, with this version requiring symptoms to have been present for 12 months. ${ }^{3}$ It is likely that this duration of problems identified, perhaps for the first time, a specific cohort of people who had long-standing issues that probably predated their time in palliative care. These data may have been different should the original version of Rome III or the more recent version of the Rome criteria (Rome IV) have been used with both these versions, requiring people to have had problems for six months with diagnostic criteria fulfilled for at least three months before presentation. ${ }^{11}$

Although this study has sought to examine factors that contribute to the severity of people's constipation symptoms, it is a secondary analysis of an existing dataset and additional research is required to explore these findings further. In addition, the duration of the time that people had been prescribed regular opioids was not included in the baseline data collection, with this factor requiring attention in the future. This is particularly when the adverse effects of prolonged opioid therapy are considered, such as pharmacologic opioid tolerance resulting in the need for dose escalation. ${ }^{18}$ Despite these limitations, a significant strength of this analysis is that it is one of the first attempts to define whether there are factors that impact the severity of a palliative patient's constipation symptoms. As many of the included factors are unlikely to be reversible, standard constipation treatments are unlikely to be effective and alternative palliative symptom management approaches are required.

\section{Acknowledgment}

This work was funded by an Australian National Health and Medical Research Council (NHRMC) grant (APP1010096).

\section{Author Disclosure Statement}

None of the authors have conflicting interests to declare.

\section{References}

1. Laugsand EA, Kaasa S, de Conno F, et al.: Intensity and treatment of symptoms in 3,030 palliative care patients: A cross-sectional survey of the EAPC Research Network. J Opioid Manag 2009;5:11-21.

2. Clark K, Smith JM, Currow DC: The prevalence of bowel problems reported in a palliative care population. J Pain Symptom Manage 2012;43:993-1000.

3. Choung RS, Locke R, Rey E, et al.: Factors associated with persistent and non-persistent chronic constipation, over 20 years. Clin Gastroenterol Hepatol 2012;10:494-500.

4. Australian Institute of Health and Welfare Palliative Care Medications: www.aihw.gov.au/palliative-care/relatedmedications/ (Last accessed July 16, 2016).

5. Mugie SM, Benninga MA, Di Lorenzo C: Epidemiology of constipation in children and adults: A systematic review. Best Pract Res Clin Gastroenterol 2011;25:3-18.

6. Dukas L, Willett WC, Giovannucci EL: Association between physical activity, fiber intake, and other lifestyle variables and constipation in a study of women. Am J Gastroenterol 2003;98:1790-1796.

7. Rosti G, Gatti A, Costantini A, et al.: Opioid-related bowel dysfunction: Prevalence and identification of predictive factors in a large sample of Italian patients on chronic treatment. Eur Rev Med Pharmacol Sci 2010;14:10451050 .

8. Abernethy AP, Shelby-James T, Fazekas BS, et al.: The Australia-modified Karnofsky Performance Status (AKPS) scale: A revised scale for contemporary palliative care clinical practice. BMC Palliat Care 2005;4:7.

9. Palliative Care Adult Network Guidelines On line Opioid Dose Converter: http://book.pallcare.info/index.php?op= plugin\&src=opiconv (Last accessed August 26, 2016).

10. Bercovitch M, Waller A, Adunsky A: High dose morphine use in the hospice setting. A database survey of patient characteristics and effect on life expectancy. Cancer 1999; 86:871-877.

11. Lacy BE, Mearin F, Chang L, et al.: Bowel disorders. Gastroenterology 2016;150:1393-1407. 
12. Slappendel R, Simpson K, Dubois D, Keininger DL: Validation of the PAC-SYM questionnaire for opioid-induced constipation in patients with chronic low back pain. Eur J Pain 2006;10:209-217.

13. Marquis P, De La Loge C, Dubois D, et al.: Development and validation of the Patient Assessment of Constipation Quality of Life questionnaire. Scand J Gastroenterol 2005; 40:540-551.

14. De Giorgio R, Ruggeri E, Stanghellini V, et al.: Chronic constipation in the elderly: A primer for the gastroenterologist. BMC Gastroenterol 2015;15:130.

15. Bell TJ, Panchal SJ, Miaskowski C, et al.: The prevalence, severity, and impact of opioid-induced bowel dysfunction: Results of a US and European patient survey (PROBE 1). Pain Med 2009;10:35-42.

16. Prichard D, Bharucha A: Management of opioid-induced constipation for people in palliative care. Int J Palliat Nurs 2015;21:272, 274-280.
17. Coyne KS, Sexton C, LoCasale RJ, et al.: Opioid-induced constipation among a convenience sample of patients with cancer pain. Front Oncol 2016;6:131.

18. Panchal SJ, Müller-Schwefe P, Wurzelmann IJ: Opioidinduced bowel dysfunction: Prevalence, pathophysiology and burden. Int J Clin Pract 2007;61:1181-1187.

Address correspondence to: Katherine Clark, MB, BS, MMed, FRACP

Department of Palliative Care Calvary Mater Newcastle

Edith Street

Newcastle 2298

Australia

E-mail: katherine.clark@calvarymater.org.au 